\title{
Penetrative convection in stratified fluids: velocity and temperature measurements
}

\author{
M. Moroni and A. Cenedese \\ Department of Hydraulics, Transportations and Roads, University of Rome "La Sapienza", Rome, Via Eudossiana 18 - \\ 00184, Italy
}

Received: 30 August 2005 - Revised: 23 May 2006 - Accepted: 24 May 2006 - Published: 7 August 2006

Part of Special Issue "Turbulent transport in geosciences"

\begin{abstract}
The flux through the interface between a mixing layer and a stable layer plays a fundamental role in characterizing and forecasting the quality of water in stratified lakes and in the oceans, and the quality of air in the atmosphere. The evolution of the mixing layer in a stably stratified fluid body is simulated in the laboratory when "Penetrative Convection" occurs. The laboratory model consists of a tank filled with water and subjected to heating from below. The methods employed to detect the mixing layer growth were thermocouples for temperature data and two image analysis techniques, namely Laser Induced Fluorescence (LIF) and Feature Tracking (FT). LIF allows the mixing layer evolution to be visualized. Feature Tracking is used to detect tracer particle trajectories moving within the measurement volume. Pollutant dispersion phenomena are naturally described in the Lagrangian approach as the pollutant acts as a tag of the fluid particles. The transilient matrix represents one of the possible tools available for quantifying particle dispersion during the evolution of the phenomenon.
\end{abstract}

\section{Introduction}

Penetrative convection is the motion of a vertical turbulent plume or dome into a fluid layer of stable density and temperature stratification when the plume has enough momentum to extend into that fluid layer for a significant distance from the original interface. In its initial stages, convection is organized in coherent structures persisting over time. Subsequently the flow becomes turbulent and the structures break up (Deardorff et al., 1969). Penetrative convection is of importance in several areas of geophysical fluid dynamics, most notably in the lower atmosphere, the upper ocean, and lakes, i.e. fluid bodies characteristically stably stratified (their mean

Correspondence to: M. Moroni

(monica.moroni@uniroma1.it) density decreases upwards) in most regions and for most of the time.

In most lakes, turbulent convective flow can be observed if the free surface is cold, which then erodes the stable stratification of the underlying lake waters on a daily or seasonal time scale (Imberger and Ivey, 1991). In the ocean under calm conditions, the upper twenty or thirty meters usually exhibit a continuous, moderately stable density distribution. When wind begins to blow over the surface, turbulence in the water is generated both by the mean shear and by sporadic breaking waves. With time, the turbulent layer becomes deeper as a result of the entrainment and erosion of the turbulence by underlying denser water. Because of the relatively rapid mixing, the density distribution is approximately uniform in the upper layer, and the entrainment takes place across the interface between the turbulent and stable fluids (Kato and Phillips, 1969).

An analogous phenomenon is observed in the atmosphere when surface heating due to solar radiation results in a growing unstable layer adjacent to the ground which replaces a nocturnal inversion from below. In this case, the initially stable environment near the ground is affected by convection, and full interaction between the two regions occurs (Deardorff, 1970; Stull, 1988). The convective boundary layer can be observed only when high intensity geostrophic winds are absent.

Fig. 1 presents an idealization of the vertical profiles of $\underline{\text { mean }}$ potential temperature, $\bar{\theta}$, and convective heat flux, $\overline{\theta^{\prime} w^{\prime}}$, over the convective layer height, $z_{i}$, where the heat flux presents a minimum. For incompressible fluids, such as water, temperature may be used rather than potential temperature. The profiles provide a guide to divide the atmospheric boundary layer into characteristic zones for penetrative convection, namely the superficial layer at the ground, the mixing layer, the transition layer and, finally, the stable layer above the convective layer, as indicated by the labels in Fig. 1. In the mixing layer with a typical height

Published by Copernicus GmbH on behalf of the European Geosciences Union and the American Geophysical Union. 


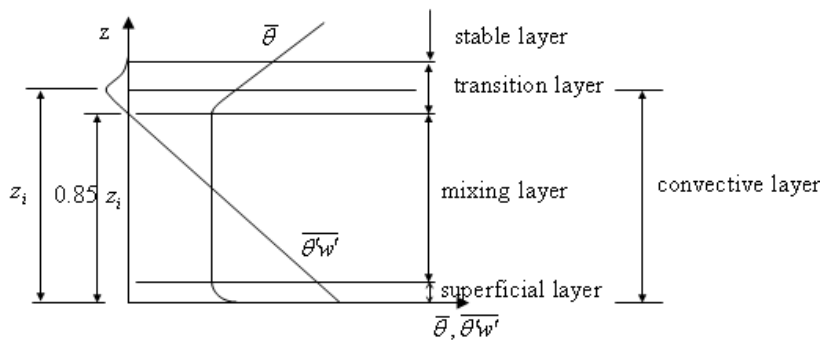

Fig. 1. Schematization of the atmospheric boundary layer under convective condition, with profiles of the vertical kinematic heat flux, $\overline{\theta^{\prime} w^{\prime}}$, and mean potential temperature, $\bar{\theta}$.

of about $0.85 z_{i}$, the potential temperature, density, and kinetic energy profiles are constant in space due to the vertical mixing of all these quantities. In the transition layer, at around $0.85 z_{i}<z<1.2 z_{i}$, the temperature profile increases and reaches a stable behavior, while the convective heat flux reaches a minimum. The mixing layer grows within the transition layer. In the stable layer, where the temperature shows a stable profile with a constant gradient, the convective heat flux is zero. It represents the boundary between the atmospheric boundary layer and free atmosphere.

At first, convection is organized in coherent structures but later the flow becomes turbulent. In the atmosphere, the convection is characterized by relatively narrow plumes in the form of domes of rising horizontal surfaces balanced by larger regions of descending motion. In lakes and oceans, an analogous phenomenon occurs but in the opposite direction; domes with large downward velocities are originating at the free surface, balanced by ascending domes with lower velocity but a larger area.

A fluid particle belonging to the mixing layer and subject to a constant buoyancy force may increase its velocity moving upward (in the atmosphere) or downward (in the lakes or oceans) to such a degree that it can penetrate into the stable layer (Stull, 1988). Resulting oscillatory movements (internal waves) generated within the stable layer take place at or below the Brunt-Väissälä frequency which is related to the vertical temperature gradient.

As the process occurs in the continually evolving mixing layer, any data which will be acquired at different times of its evolution will have to be normalized to eliminate the time dependence. The similarity proposed by Deardorff (1970) was employed here to compute scaling parameters, which in themselves are functions of time. The convective scaling assumes that the mechanical production of turbulence kinetic energy is negligible in comparison with buoyancy production. Under this assumption, the scaling parameters are:

height of the mixing layer: $z_{i}$

convective velocity: $w_{*}=\sqrt[3]{g \beta q_{s} z_{i}}$

convective time: $t_{*}=\frac{z_{i}}{w_{*}}$

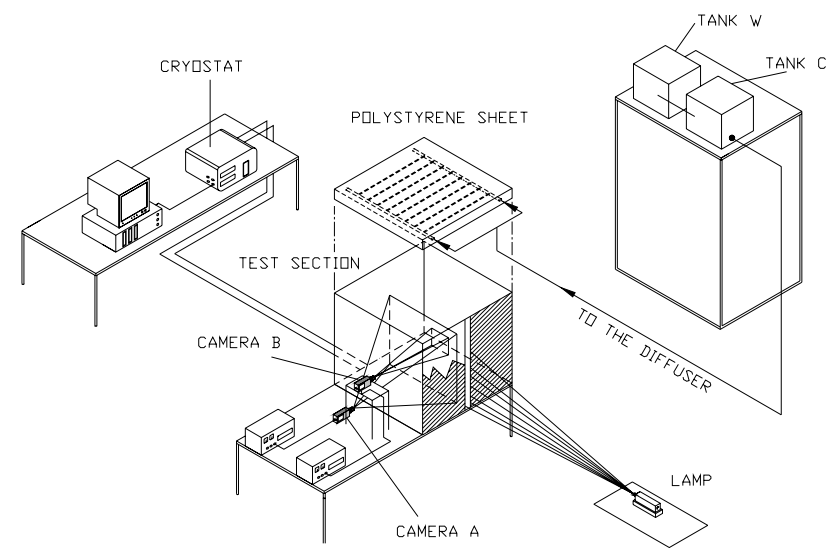

Fig. 2. Experimental set-up.

where $q_{s}$ is the surface kinematic heat flux $\left(\overline{w^{\prime} \theta^{\prime}}\right.$ at the boundary) and $\beta$ the volume expansion coefficient. Through normalizing the quantities measured at different stages of the experiment, the phenomenon can be considered as a succession of steady states, according to an evolution of the quantities of interest that we may define quasi-steady (Cenedese and Querzoli, 1994).

Several numerical simulations were developed to investigate penetrative convection (Nieuwstadt et al., 1992; Klemp et al., 2000) but few laboratory simulations have been published. Townsend (1964) presented an experimental investigation where a tank of water, initially isothermal, was cooled from below and heated gently from above. An unstable layer developed adjacent to the bottom of the tank when a temperature lower than $4^{\circ} \mathrm{C}$ (temperature of maximum density) was reached. As it thickened, a stable layer developed above it. The temperature fluctuations were of maximum amplitude at the level of the mean interface between the lower unstable and upper stable layers, or slightly above it. In the upper stable region, these fluctuations appeared to be associated with internal waves excited by the impact of columns penetrating from below and subsequently subsiding. It should be noted that this experiment is fundamentally different than the atmospheric case where the heat flux gradient is nearly constant with height.

Deardorff et al. (1969) presented an experiment where the stratification of the stable layer was distributed continuously and uniformly throughout the layer, and the energy input was by heat flux at one boundary. The resulting mean thermal structure was steady and the convective layer continually deepened, where the stratification within the stable layer remained essentially constant while the interface is eroded. The horizontally averaged temperature was found to vary smoothly with height and to undergo a slight cooling just above the inversion base (i.e., depth of the mixed layer). The maximum cooling was related to the downward heat transport at the inversion base and to the rise rate of the latter. The 


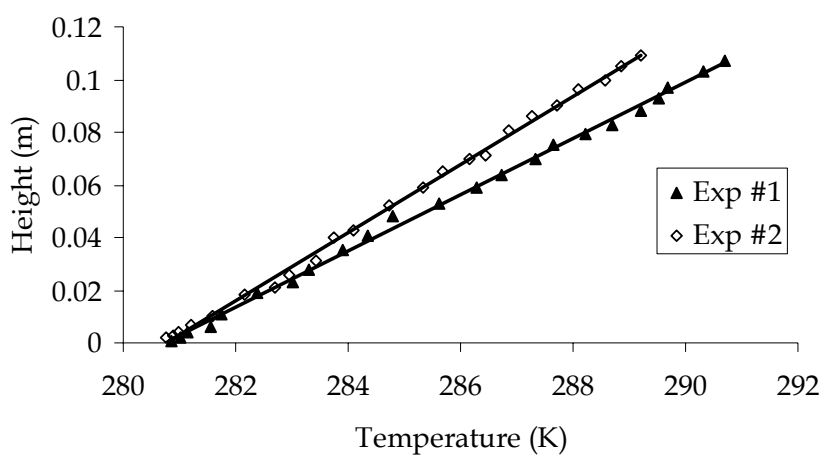

Fig. 3. Final stratification profiles.

local interface between the stable region and the convective region remained well defined throughout the experiments and was found to be a combination of: domes with adjacent cusps through which downward entrainment occurred, flat sections, folded structures and breaking waves.

A laboratory model of the Convective Atmospheric Boundary layer consisting of a convection chamber was developed by Cenedese and Querzoli (1994). The thermal structure and the turbulence statistical moments were investigated using temperature measurements from a thermocouple and velocity data from a Laser-Doppler anemometer (LDA). Using the Convective Boundary Layer scaling from Eq. (1), turbulent heat-flux, vertical velocity and temperature variance, and probability distributions were computed. Autocorrelation and spectra were evaluated as well.

Querzoli (1996) presented simulations of turbulence in the unstable boundary layer of the atmosphere by means of a laboratory model using non-intrusive Particle Tracking Velocimetry (PTV) that allowed the investigation of particle dispersion in a Lagrangian framework. From the Lagrangian correlations of the horizontal and vertical velocity components, the Lagrangian integral time scales were obtained and compared to the Eulerian measures. The data set was conditionally sampled to describe selectively the behavior of upward and downward moving particles. The comparison between Lagrangian and Eulerian time scales showed the impossibility of defining a single value of their ratio for the whole unstable boundary layer.

Cenedese and Querzoli (1997) presented the application of PTV to pollutant dispersion in a laboratory simulation of the atmospheric convective boundary layer. The convective layer was simulated by a water tank heated from below, where the atmospheric thermal stratification was reproduced. The pollutant dispersion was described by the transilient matrix representing the probability of transition of a particle from one level to another of the convective layer.

The work presented here contributes to this research in three ways. The combined use of thermocouples and flow visualization techniques has, for the first time, allowed the simultaneous measurement of temperature and velocity com-
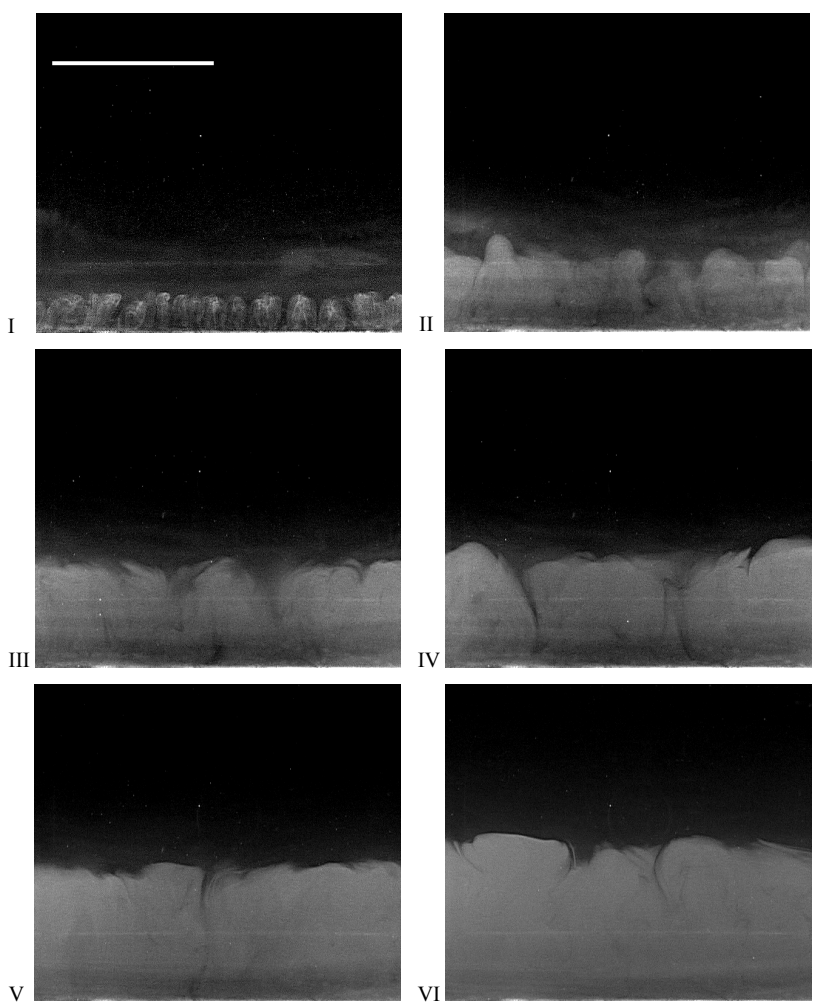

Fig. 4. Visualization of the mixing layer evolution. Each snapshot corresponds to a progressive time (the white line in frame I is $10 \mathrm{~cm}$; I: 180 s; II: 400 s; III: 600 s; IV: 700 s; V: 919 s; VI: 1260 s)

ponents. This then allowed to employ and cross-validate different methods to estimate the mixing layer growth. Thirdly, Feature Tracking (FT) was used to analyze velocities rather than the more traditional Particle Tracking Velocimetry which is limited to a relatively low particle density.

The following two sections introduce the experimental setup and Feature Tracking, respectively. Section 4 introduces qualitative results using Laser-induced fluorescence (LIF) and quantitative flow field results from Feature Tracking, while Sect. 5 presents the analysis of the mixing layer height and a measure of mixing using the transilient matrix. A brief discussion and summary concludes this paper in Sect. 6 .

\section{Experimental set-up}

\subsection{The apparatus}

The container, as shown in Fig. 2, is a prismatic tank of square cross-section, $41 \times 41 \times 40 \mathrm{~cm}^{3}$. Its sidewalls are insulated by $3 \mathrm{~cm}$ thick polystyrene sheets. Insulation is maintained while the tank is filled with the density-stratified fluid but removed at the start of the experiment on the side facing the camera, to allow access for viewing. 


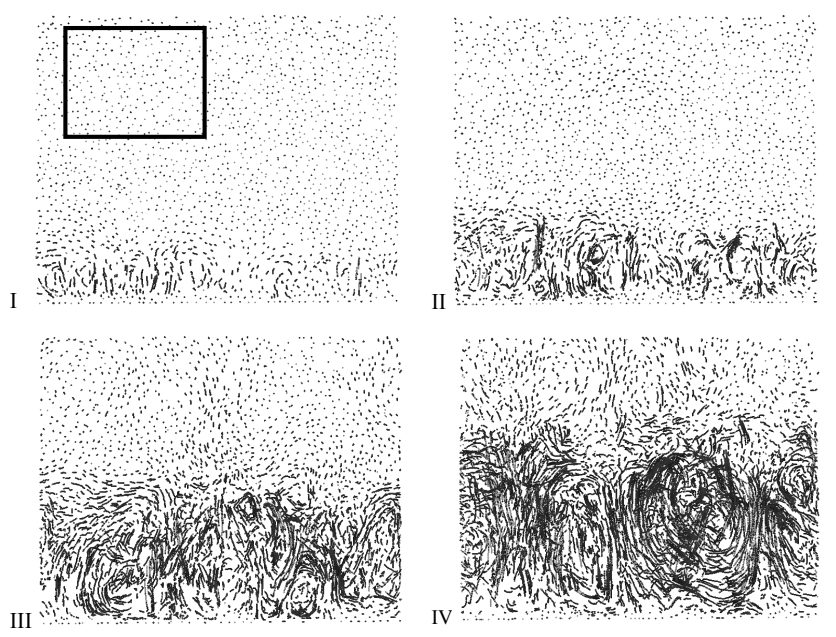

Fig. 5. Features and trajectories reconstructed by FT during the mixing phenomenon using Camera A (exp \#2; I: 130 s; II: $260 \mathrm{~s}$; III: $600 \mathrm{~s}$; IV: $1100 \mathrm{~s}$ ). The rectangle shows the region captured by Camera B.

The fluid within the test section is in contact with a circulating water bath on its lower boundary, separated from the fluid by a horizontal aluminum sheet fitting the tank horizontal cross-section. A cryostat is connected to this circulating water bath to control the temperature of the lower boundary. During the filling process, this temperature is set to maintain the stable stratification, but is then increased to start and maintain the convection by maintaining a temperature always greater than the average temperature within the mixing layer.

\subsection{Measurement equipment}

Temperature measurements were taken with thermocouples placed within the test section along a vertical line (array of 27 thermocouples) to measure vertical profiles and also in a horizontal line on the lower boundary to test horizontal homogeneity.

Some visualization experiments with dye were performed to visualize the movement and shape of the interface between the stable and unstable layers. In those cases, fluorescein was placed along the lower boundary of the test section.

The velocity field in a vertical cross-section was obtained by Feature Tracking. Images of highly reflective tracers (pollen particles with average size of about $=80 \mu \mathrm{m}$ ) were recorded using two monochrome 8-bit CCD cameras with a time resolution of $25 \mathrm{fps}$, one focused on the mixing layer region (Camera $\mathrm{A}$ - large acquisition window of about $15 \mathrm{~cm}$ width) and the other on the internal waves area (Camera B - smaller acquisition window of about $5 \mathrm{~cm}$ width). The fluid was seeded during the filling procedure of the test section. The measuring volume was illuminated by a $1000 \mathrm{~W}$ arc lamp, and a beam stop allowed the depth of the illuminated area to be controlled.
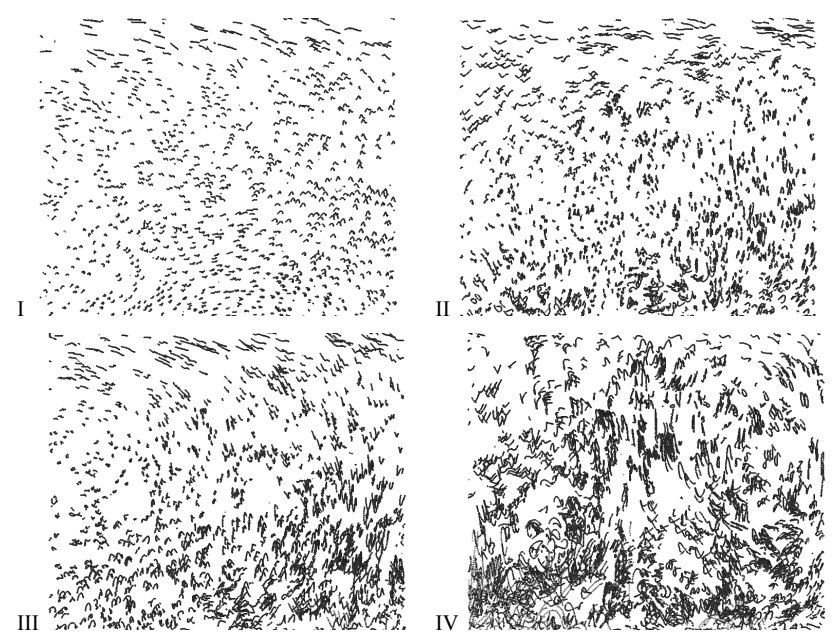

Fig. 6. Features and trajectories reconstructed by FT within the stable layer (exp \#2; I: 200 s; II: 560 s; III: 600 s; IV: 800 s).

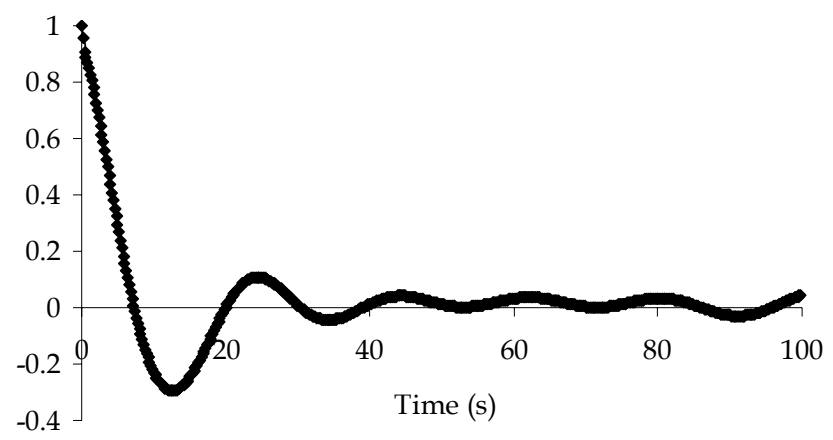

Fig. 7. Correlation coefficient for w for experiment \#2.

The acquisition procedure can be divided into three steps: at first images were recorded on a tape, then the images were digitized with a resolution of $576 \times 720$ pixels and temporarily stored on the mass memory of a computer, and finally they were analyzed to identify features and follow their trajectories. This final step is described in a little more detail in Sect. 3.

\subsection{The working fluid and filling procedure}

The working fluid is distilled water which is initially stably stratified by a uniform vertical temperature gradient. Water is used, rather than air, to allow both a large heating rate and sufficient time to take measurements of the changing thermal structure. However, the similarity with the phenomenon

occurring in the atmosphere still holds.

The stable stratification within the test section (i.e, a positive vertical temperature gradient) is obtained by the two tanks method. The fluid is initially equally distributed into two connected identical tanks set at the same height. The temperatures inside the tanks are initially set to $T_{w}$ in the 


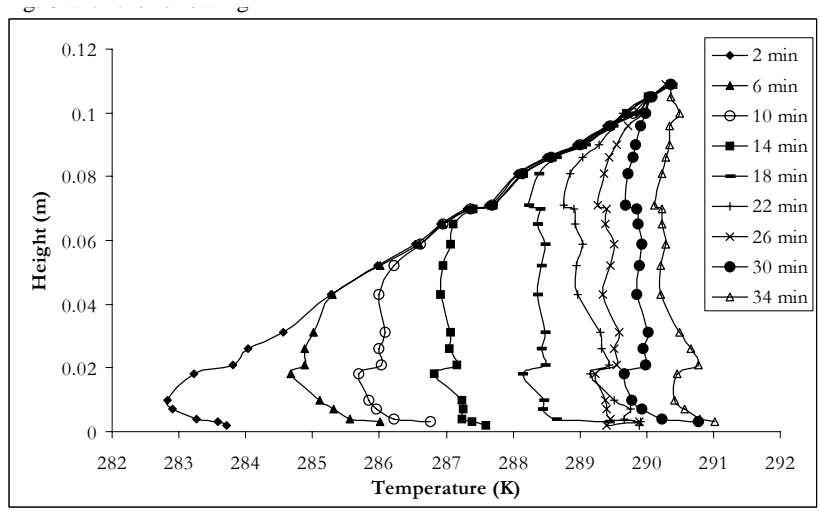

Fig. 8. Temperature vertical profiles for experiment \#2.

"warm" tank and $T_{c}$ in the "cold" tank. The test section is filled through a diffuser placed behind a polystyrene sheet connected to a tube from the cold tank. As water from the cold tank drains into the test section, the water level in the tanks is equalized by water flowing from the hot tank to the cold tank, thus gradually increasing the temperature in the cold tank. An agitator is placed within the cold tank to ensure a uniform temperature within that tank.

During the filling procedure, the temperature at the upper boundary of the test section is maintained at a constant value by the insulating polystyrene sheet. At the lower boundary, the cryostat maintains the temperature at a fixed value, approximately equal to the cold tank temperature.

\subsection{Initial conditions and convection experiments}

The initial fluid conditions are zero velocities while the temperature increases with height from the lower boundary temperature, $T_{b 0}$, with an approximately linear trend of slope $\alpha$. Figure 3 displays the temperature distribution from the vertical thermocouple array for two experiments discussed here before heating was started. The symbols represent the measurements and the lines the linear regressions to the data with the gradients listed in Table 1.

Table 1 presents typical values for the two experiments described below, where the slope was found from the thermocouple measurements by linear regression and $T_{b 0}$ is the measured bottom temperature from the linear regression as well. The Brunt-Väissälä frequency, $N\left[\mathrm{~s}^{-1}\right]$, also listed in Table 1 , is computed from

$N=\left(g \beta\left(\frac{\partial \bar{T}}{\partial z}\right)\right)^{1 / 2}$

Thermal convection is initiated upon replacing the cool water circulating under the lower boundary with warm water of temperature greater than the upper boundary temperature (time $t=0$ ).

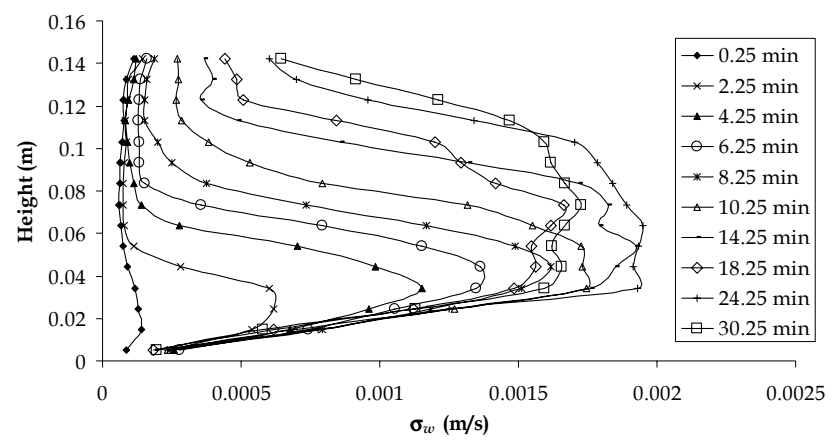

Fig. 9. Standard deviation profiles of the velocity vertical component for experiment \#2.

Table 1. Initial conditions of the experiments.

\begin{tabular}{llll}
\hline Experiment \# & $\begin{array}{l}T_{b 0} \\
(\mathrm{~K})\end{array}$ & $\begin{array}{l}\alpha=(\partial \bar{T} / \partial z) \\
(\mathrm{K} / \mathrm{m})\end{array}$ & $\begin{array}{l}N \\
\left(\mathrm{~s}^{-1}\right)\end{array}$ \\
\hline 1 & 280.8 & 93.4 & 0.35 \\
2 & 280.7 & 77.1 & 0.30 \\
\hline
\end{tabular}

\section{Velocity measurement through Feature Tracking (FT)}

Feature Tracking, rather than the classical Particle Tracking Velocimetry (PTV), was used to reconstruct tracer particle trajectories for two reasons. For one, PTV is limited to low particle density images. The second reason was that FT does not require a known background flow or a priori velocity estimates to identify particles and their trajectories (Udrea et al., 2000; Cenedeseet al., 2002). The technique employed for this investigation implemented a pure translation model (Miozzi, 2004; Moroni and Cenedese, 2005). FT and PTV share the image acquisition steps, namely seeding the flowing fluid with small highly reflecting particles to a given tracer particle density, illuminating the flow field with a light sheet, and acquiring images of the particles located in the sheet with an imaging rate that determines the time resolution. The techniques then diverge in the analysis of these images.

An image, $I$, is defined as a set of positive integer values from 0 to $2^{\text {nbits }}-1$, where "nbits" is the number of bits of the signal from the sensor (the camera), defined on a compact, discrete support $\Omega$ (the sensor matrix). $\Omega$ is discretized on a regular grid of $M \times N$ elements (pixels). The value assigned to each pixel in an image $I$ represents the 2-D projection of the 3-D world onto the sensor plane. Here, it is assumed that all surfaces inside each image have Lambertian characters (i.e., their luminosity values do not depend on the point of view of the observer) and that the illumination source gives almost constant light levels. 


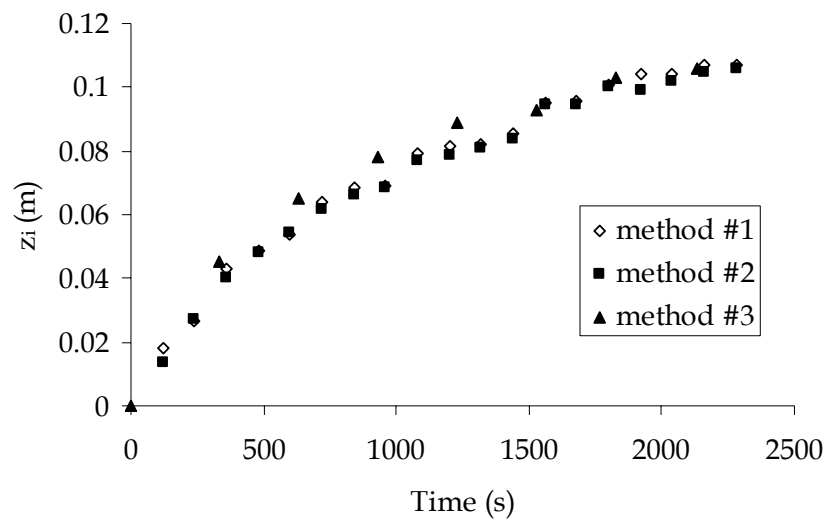

Fig. 10. Comparison of mixing layer growth with the standard deviation method and temperature method for experiment \#2.

The Feature Tracking algorithm identifies intensity gradients within each image as features and tracks them from frame to frame. Intensity gradients may be located within the image background, on the tracer particles, or all around their boundary. A threshold for the intensity gradient is introduced to take into account the noise inside images and to reject features in the background. If particle size is small, features tracked by the algorithm are likely to be located around the particles boundaries.

Once a feature has been identified, a window, $W=L \times H$, of length $L$ and height $H$, is constructed around the feature and centered at its location. The dissimilarity between the sub-image $I_{W}$ at time $t_{A}\left(I_{W A}\right)$ and at a successive time $t_{B}=t_{A}+\Delta t\left(I_{W B}\right)$ is called Sum of Squared Differences (SSD) (Lukas and Kanade, 1981; Tomasi and Kanade, 1991). The location of $\left(I_{W B}\right)$ that minimizes SSD provides the displacement of the feature under investigation. In particular, the velocity vector is computed by dividing the distance between the centroids of $I_{W A}$ and $I_{W B}$ by the time interval. In the purely translational motion model, the motion is assumed to be constant in the interrogation region $W$ ("frozen" hypothesis).

The feature tracking algorithm requires the input of the following parameters:

- minimum distance among features. This must be chosen according to image seeding density. It has no effect on the trajectories reconstructed, but only determines the quantity of features to track, and, as a consequence, the number and density of the velocity vectors.

- window size. This parameter influences tracking efficiency. If the image seeding density is high, large windows will include a large number of particles providing an averaged velocity vector instead of the velocity of the particle under investigation. On the other hand, small windows may determine the computation of an unreliable dissimilarity.
Table 2. Comparison between internal wave $(\omega)$ and Brunt-Väissälä (N) frequency.

\begin{tabular}{lll}
\hline Experiment \# & $\begin{array}{l}N \\
\left(\mathrm{~s}^{-1}\right)\end{array}$ & $\begin{array}{l}\omega \\
\left(\mathrm{s}^{-1}\right)\end{array}$ \\
\hline 1 & 0.35 & 0.33 \\
2 & 0.30 & 0.26 \\
\hline
\end{tabular}

\section{Flow visualization results}

In this section, some qualitative results from a LIF experiment and the quantitative results from the FT analysis of the two experiments listed in Table 1 are presented.

\subsection{Mixing layer evolution}

Figure 4 shows images of the mixing layer and its interface. Images were acquired during an experiment when fluorescein was placed at the bottom of the test section. A camcorder was used to acquire images. The portion viewed in the figures is a vertical slab about $1 \mathrm{~cm}$ thick centered near the middle of the test section. In general, the interface shape appears quite complicated. The evolution of the mixing layer can be qualitatively observed. In frame I, $180 \mathrm{sec}$ after starting the heating, domes of fairly regular cross-section of about $18 \mathrm{~mm}$ and height of about $25 \mathrm{~mm}$ have formed. In frame II, $220 \mathrm{sec}$ later, the mixing layer has grown to a height of $47 \mathrm{~mm}$, and plumes or domes have merged to plumes of cross-sections from 32 to $50 \mathrm{~mm}$. The broadening of the plumes continues in frames III to VI but the growth of the mixing layer height slows down, with heights of about $62 \mathrm{~mm}$ and $100 \mathrm{~mm}$ after $600 \mathrm{sec}$ and $1260 \mathrm{sec}$ after the beginning of the experiment, respectively.

Figure 5 displays features and their trajectories reconstructed by FT for exp \#2 inside both the stable and the unstable layers as they evolve with time (Camera A). In each picture, features and corresponding trajectories reconstructed over 250 consecutive frames are overlaid, resulting in trajectories extending over a time interval of $10 \mathrm{sec}$. Small segments characterize particles moving with a small velocity while long segments characterize faster particles; identical trajectory lengths correspond to identical velocities and direct picture-to-picture comparisons can be made.

The stable layer, with vanishing velocities and small tracer displacements, can be clearly seen, as can the developing convective layer below. The depth of the convective region and the length scales are consistent with those seen in the LIF images in Fig. 4, but one can additionally see in frames III and IV that some fluid motion is initiated within the stable layer. The convective motions grow, starting from the lower warm boundary and giving rise to upward domes. They coalesce, forming larger structures with higher velocity. After a 


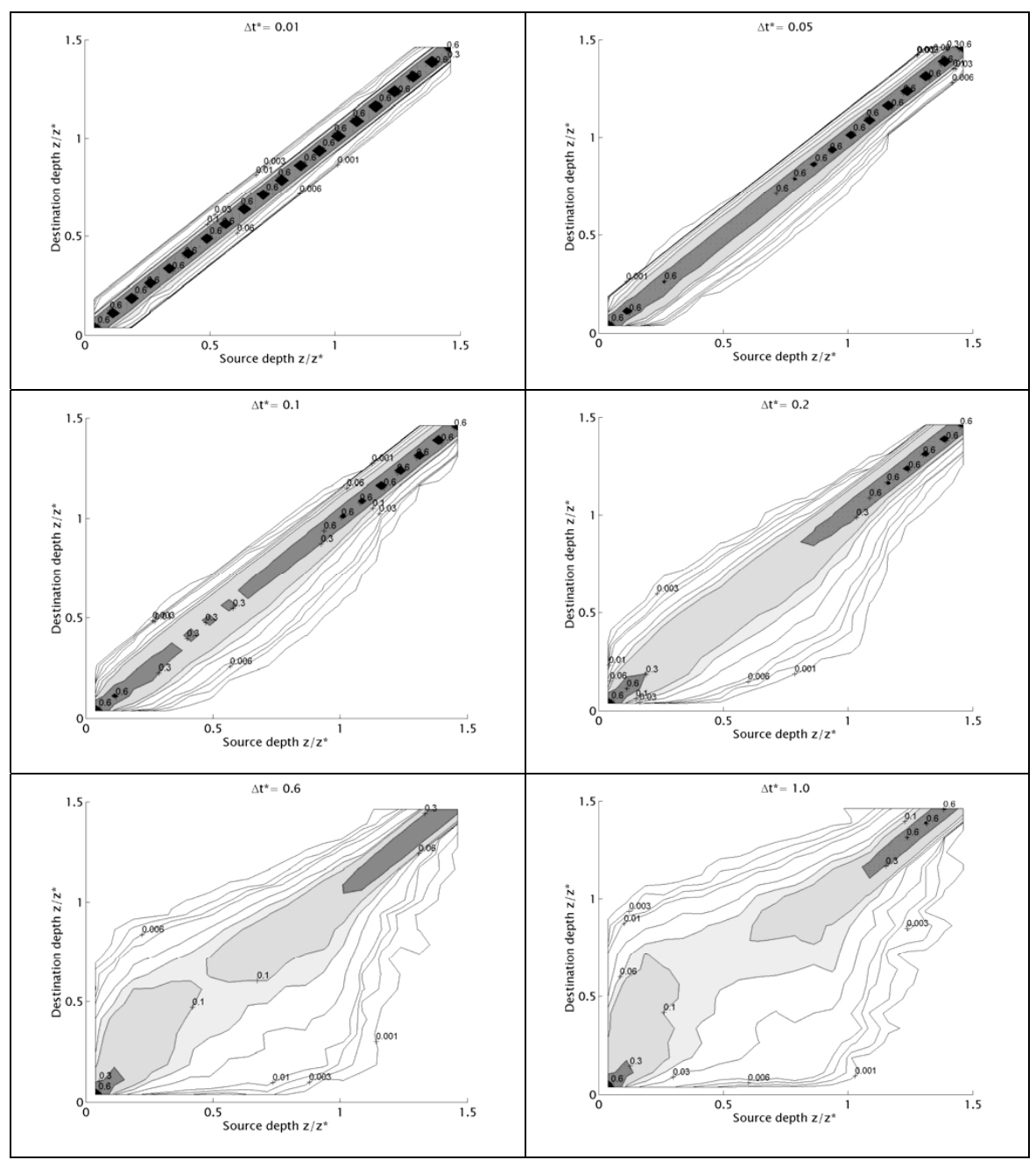

Fig. 11. Contour plots of the evolving transilient matrix for experiment \#2. The contour levels are 0.001, 0.003, 0.006, 0.03, 0.1, 0.3, 0.6.

particle leaves the boundary, it travels upwards until the stable layer is reached. Fluid particles belonging to the stable layer are later entrained within the mixing fluid. The stress the mixing layer produces on the stable layer becomes more important as time goes on, causing increasing amplitude of the internal waves.

\subsection{Internal waves evolution}

Figure 6 presents features and trajectories belonging to the stable layer reconstructed by FT (Camera B). The interaction of the domes with the stable layer produces the oscillation of tracer particles belonging to the stable fluid (internal waves). The oscillatory movement starts in the fluid volume close to the mixing layer and moves upward with time. The same particles describing oscillating trajectories are later entrained inside the mixing fluid of increasing height. Internal waves will then be present over the entire stable layer. The oscillation amplitude increases with time, increasing the energy input from the mixing layer. The oscillation frequency of internal waves, $\omega$, cannot be greater than the Brunt-Väissälä frequency, $N$. To test whether the measured velocities are due to internal gravity waves, Fig. 7 presents the Lagrangian correlation coefficient of the vertical velocity component $(w)$ for Experiment\#2. The distance between two consecutive peaks of the correlation coefficient provides the internal wave oscillation period, $T=2 \pi / \omega$. Table 2 presents the comparison between the frequency of the internal waves, $\omega$, and the Brunt-Väissälä frequency, $N$, respectively. As expected, $\omega$ of the same order of magnitude but lower than $N$ in both cases.

\section{Mixing layer growth and transport features}

In this section, the temperature and velocity data will be used first to obtain three independent quantitative results for the mixing layer height and then to estimate transport characteristics using the transilient matrix. 


\subsection{Mixing layer growth}

The accurate detection of $z_{i}(t)$ is a preliminary step to gather meaningful information from the Lagrangian analysis of the dispersion phenomenon occurring within the mixing layer. To obtain the mixing layer height as accurately as possible, and with a measure of the reliability of the result, three independent methods were employed to calculate the mixing layer height and its evolution, $z_{i}(t)$. The first method is based on identifying the three basic sections of the temperature profile as illustrated in the idealized profile of $\bar{\theta}$ in Table 1 . The second is based on vertically averaged temperature measurements, and knowledge of the initial stratification. The third method, finally, is based on the velocity data.

\subsubsection{Method 1, based on temperature profiles}

Vertical temperature profiles allow the measurement of the growth of the mixing layer with time. When we start heating from below, the temperature profile changes with time as far as the phenomenon evolves (Fig. 8). Three characteristic portions characterize each profile. The portion of the profile close to the boundary presents a negative gradient related to the existence of the thermal boundary layer. The profile then has a uniform temperature where the mixing layer is located. Finally above the mixing region, the temperature profile practically collapses onto the straight line of the initial stratification. Each temperature profile in Fig. 8 is associated with the acquisition time given in the legend even though it was obtained through averaging temperature data acquired for $30 \mathrm{sec}$ at each thermocouple location. We could then construct an idealized profile by drawing the profile from the linear regression and a vertical line with the average temperature of the mixing layer. The intersection between the two lines identifies the mixing layer height associated to that profile. This method relies on having enough temperature measurements in both the mixing layer and the stable layer to construct a profile at each time but it does not rely on any prior information such as the initial conditions.

\subsubsection{Method 2, based on the initial stratification}

If the initial condition is known, as it was in our experiments, with $T(z)=T_{b 0}+\alpha z$ where the constants are listed in Table 1 , one can also calculate the mixing layer height from this initial profile and the measured temperature profile at time $t$. Since the temperature in the mixing layer is constant and equal to that at the top of the mixing layer, the temperature within the mixing layer, $\bar{T}(t)$, is related to $z_{i}(t)$ through the following relation:

$z_{i}(t)=\frac{1}{\alpha}\left(\bar{T}(t)-T_{b 0}\right)$.

Knowing the temperature within the mixing layer only, the height can therefore be calculated. While this method 2 relies on knowing the stable stratification, either from measure- ments of the initial conditions for the experiment, or from field measurements at another time, it does not require any measurements from outside the mixing layer.

\subsubsection{Method 3, based on velocity data}

The third method to detect the mixing layer growth consists in reconstructing the vertical velocity component standard deviation profile as a function of time employing the horizontal homogeneity hypothesis. The mixing layer is characterized by greater values of the standard deviation than the stable layer.

Figure 9 presents the profiles of the vertical velocity component standard deviation $\left(\sigma_{w}\right)$ at several times. At the beginning, the standard deviation is very small everywhere but an indication of the onset of convection can be seen close to the lower boundary. As time goes on, the standard deviation increases in magnitude and covers an increasing portion of the fluid. This is a reflection of the growing mixing layer characterized by great fluctuations of the velocity field about the mean value. The standard deviation profile shows a sharp increase from the boundary with height, where the slope of that increase appears to be constant over time at about $17 \mathrm{~s}$. It is then for a substantial part of the evolution characterized by a narrow maximum well within the mixing layer followed by a decrease with a slope of $36 \mathrm{~s}$. At later stages in the evolution, the standard deviation appears to saturate and a region of relatively uniform standard deviation characterizes the bulk of the mixing layer. Using these standard deviation profiles, the mixing layer height, $z_{i}(t)$, was calculated. In all cases, the transition from the mixing layer to the stable layer, i.e. the mixing layer height, is shown to be the termination of the decreasing slope of the standard deviation in a relatively uniform standard deviation at small values.

\subsubsection{Mixing layer height results}

Figure 10 presents the superimposition of the mixing layer growth profiles detected. The estimate from method 3 tends to be a little higher than that from the other two methods. Methods 1 and 2 are extremely close. Since they differ only in the fact that method 1 uses the instantaneous temperature in both the unstable and the stable layers but method 2 uses the initial temperature profile, this can be taken as an indication that the temperature profile in the stable layer is not noticeably affected by the growing mixing layer. This seems to hold even at late stages in the evolution when the velocity data indicate some internal wave activity in the stable layer. Despite the small difference between methods 1 and 2 on one hand and method 3 on the other, all three methods produce a result consistent with each other.

\subsection{Transilient matrix}

The transilient matrix technique was employed for detecting non-local transport features. To describe the transport 


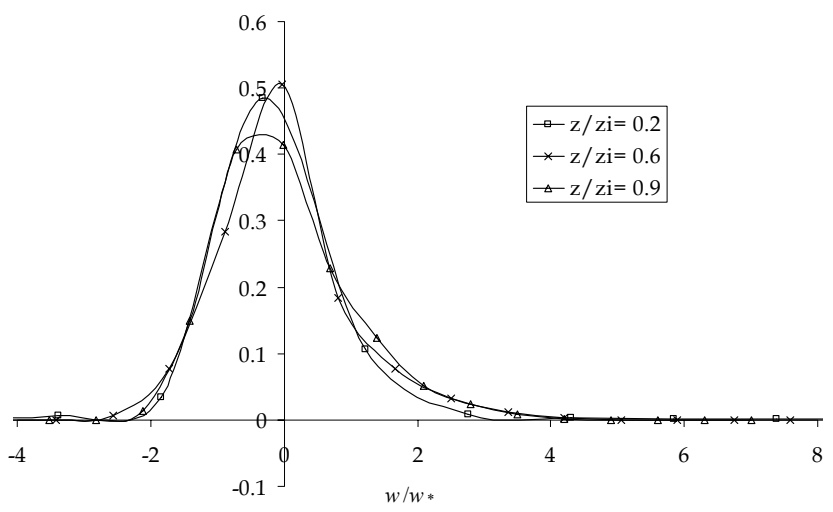

Fig. 12. Probability density distribution of the non-dimensional vertical velocity.

properties in such a stochastic non-local frame of reference, the probability density $p\left(\mathbf{X}_{1}, t_{1}, \mathbf{X}_{2}, t_{2}\right)$, i.e. the probability of particles starting at time $t_{1}$ at location $\mathbf{X}_{1}$ to reach location $\mathbf{X}_{2}$ at time $t_{2}$, has to be computed. The probability density $p$ is in general a function of eight independent variables: $t_{1}, t_{2}, \mathbf{X}_{1}$ and $\mathbf{X}_{2}$. This can be reduced if we assume the convective boundary layer to be stationary (after quantities are normalized). Considering thermal convection, it is natural to focus on transport in the vertical direction, parallel to the direction of the gravitational field. This suggests the convective layer to be considered horizontally homogeneous. The probability that a particle moves from a location of height $z_{1}$ to another location of height $z_{2}$ in a given time interval $\delta t=t_{2}-t_{1}$ can then be obtained. This statistic, named transilient matrix, is neither Eulerian, because the starting location of particles is considered, nor Lagrangian because the statistic is not computed along the trajectories. Several quantitative descriptors of non-local transport can be computed from the transilient matrices obtained for a given flow. In practice, the transilient matrix is computed from the statistics of displacement of a large number of passive tracer particles initially uniformly distributed among levels. The flow field advects these particles. The flow domain is subdivided into $N$ horizontal layers of equal thickness $\Delta z$ (bins). The location index $m$ represents a grid point within a column of equally-spaced grid cells at the center of the cell at height $z_{m}=(m-0.5) \Delta z$ where $\Delta z$ represents the vertical discretization step. In its most general definition, each element of the transilient matrix $c_{m l}(t, \Delta t)$ represents the fraction of particles advected to a destination grid cell at vertical location index $m$ from a source grid cell at location $l$ during a time interval $t$ to $t+\Delta t$. The vertical coordinates of tracer particles have been normalized by the mixing layer height. The time associated with each particle location is normalized as well by the convective time. The matrix is now a function of the non-dimensional time interval $\left(\Delta t^{*}\right)$ alone.

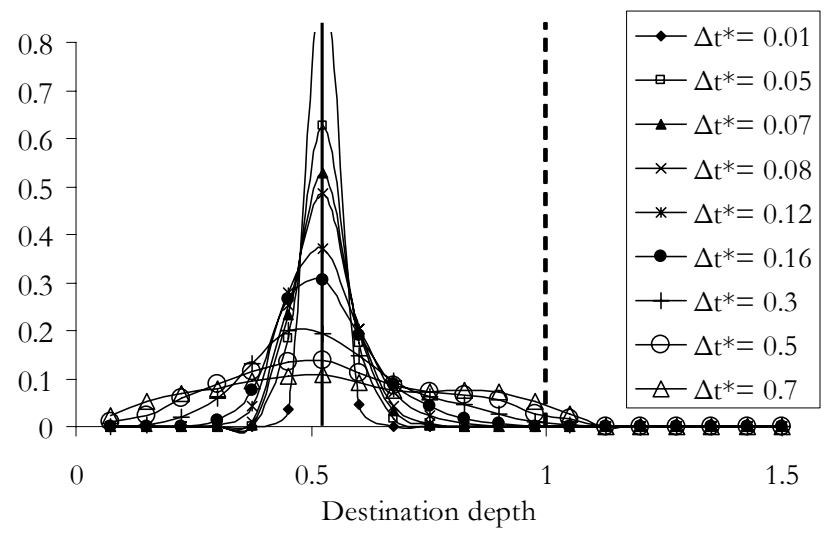

Fig. 13. Cross section through the transilient matrix at a source depth of $\mathrm{z} / \mathrm{zi}=0.525$

In general, after the action of turbulence during a time interval $\Delta t^{*}$, a destination cell $i$ should contain a set of particles originating from a variety of sources. Elements along the main diagonal of the matrix indicate the fraction of particles in cell $i$ that is not involved in turbulent mixing. This may be the case of absence of mixing to or from that cell, or may depend on $\Delta t^{*}$. If $\Delta t^{*}$ is of the same order of magnitude as the time scale of the structure involved in the mixing process, the particle position at time $t^{*}$ and its position at time $t^{*}+\Delta t^{*}$ may belong to the same layer owing a contribution to the element of the matrix along the diagonal. This may occur at the early stage of the phenomenon when domes are organized in coherent structures and particles travel along small vortices crossing upwards and downwards the mixing layer region. The phenomenon will evolve in a turbulent (i.e. disorganized) fashion. It is unlikely that particles will come back to their starting position being probably moved upwards by an arising dome.

The transilient matrices were computed taking into account particles belonging to a small portion of stable layer located close to the mixing region. The region of interest was divided into 15 sub layers of equal thickness.

The temporal evolution of the transilient matrix for experiment\#2 is exhibited in Fig. 11. Although the spatial extent of each bin increases with $\Delta t^{*}$ due to the increase of the mixing layer height, the matrices are plotted with respect to the origin and destination depths in order to simplify their physical interpretation. The destination index increases from the bottom to the top. Height will then increase upwards.

For small $\Delta t^{*}\left(\Delta t^{*}<\sim 0.05\right)$, particles will have moved only a short distance or they still belong to their starting position. The matrix, in its graphical representation, presents darker zones along the diagonal (probability close or equal to 1), corresponding to absence of mixing. For intermediate time lags $\Delta t^{*}\left(\Delta t^{*}=0.05\right.$ to 0.2$)$ the darker area starts spreading out mainly below the matrix diagonal, evidencing a slow mixing process occurring downwards, corresponding to the 
negative value of the velocity probability density function mode. For large time lags $\Delta t^{*}\left(\Delta t^{*}>\sim 0.6\right)$, particles spread out almost uniformly within the mixing layer and the transilient matrix assumes uniform values in that area. The stable layer is also evident in the graphical representation of the matrix: the reduced motion characterizing the stable layer is associated with large values of the displacement probability. Figure 12 presents the probability density distribution of the vertical velocity vs. dimensionless velocity fluctuations at three different levels. All distributions are not symmetrical with a slightly negative mode. This confirms the fact that the convective boundary layer is characterized by small and intense updraughts and large but slow downdraughts (Cenedese and Querzoli, 1997).

Figure 13 presents a cross section through the transilient matrix. The concentration begins as a $\delta$ function at the source depth, marked with a continuous line, and progressively more disperse curves correspond to later times. The dotted line marks the boundary of convection zone. If the mixing occurred via classical dispersion in an infinite domain, cross sections through the matrix would yield Gaussians which would progressively decrease in amplitude and increase in dispersion with time.

\section{Discussion and conclusions}

The flux through the interface between the mixing layer and the stable layer plays a major role in understanding, characterizing and forecasting the quality of water in stratified lakes and in the upper portion of the oceans and the quality of air in the atmosphere. These issues have motivated our experimental investigation that was aimed at predicting mixing layer growth as a function of initial and boundary conditions, understanding the interaction between the mixing layer and the stable layer (e.g., internal waves) and describing the fate of a contaminant dissolved within the fluid phase. Characteristic structures have been observed in the convective boundary layer: growing domes or turrets presenting an extremely sharp interface at their top, flat regions of large horizontal extent after a dome has spread out or receded, and cuspshaped regions of entrainment pointing into the convective fluid. Dome characteristic dimensions are of the same order of magnitude as the mixing layer height, while their lifetime is less or equal to the time a fluid particle would need to complete a whole cycle moving through the rising dome and returning in the downwelling region.

There are some phenomena, i.e. pollutant dispersion, that are naturally described in a Lagrangian frame of reference. For a full Lagrangian description, particles have to be tracked for a period of several phenomenon time scales.

In the simplest treatment of mixing, one prescribes a dispersion coefficient which scales with characteristic size and velocity (Moroni et al., 2003; Kleinfelter et al., 2005; Cushman et al., 2005). The dispersion coefficient is typically much greater than diffusivity arising from microscopic processes alone (Miesch et al., 2000). The main assumption behind the approach is locality. Referring to turbulent mixing, locality means that a particle is carried by one eddy only a short distance before being in a different eddy completely uncorrelated with the first. Locality is then associated to short correlation of the velocity field. When the hypotheses concerning locality fail, i.e. when turbulent convection occurs, a non-local description of turbulent transport has to be employed. In this context, the transilient matrix formalism represents a useful tool available for quantifying particle dispersion during the evolution of convective mixing.

The flow under investigation is unsteady in a Lagrangian reference frame since particles, during their motion, reach regions that can be considered homogeneous only in the horizontal plane. The Feature Tracking technique allows long trajectories to be reconstructed within the flow field. As images with a large particle density can be analyzed with FT, the statistics can be more robust than from classical Particle Tracking Velocimetry.

Results stress the need for a non-local generalization of the concept of classical dispersion, in which the dispersive flux of a passive scalar is assumed to be proportional to gradients in the mean concentration. The evaluation of the transilient matrix permits estimation of pollutant dispersion whatever the height of the source. Superimposing results from different heights, multiple and distributed sources can be simulated. Finally, assuming a negligible horizontal dispersion compared with transport due to a mean wind, the concentration field downwind from a source can be simulated (Cenedese and Querzoli, 1997).

Acknowledgements. The authors are grateful to A. Baldinotti, G. Barbato, M. Colò, M. De Dominicis, V. Dore, and J. Romero for their indispensable help in taking measurements. Thanks to W.G. Früh for assistance in clarifying the English and improving the quality of this work. The anonymous reviewers are acknowledged as well.

Edited by: W.-G. Früh

Reviewed by: two referees

\section{References}

Cenedese, A. and Querzoli, G.: A laboratory model of turbulent convection in the atmospheric boundary layer, Atmos. Environ., 28(11), 1901-1913., 1994.

Cenedese, A. and Querzoli, G.: Lagrangian statistics and transilient matrix measurements by PTV in a convective boundary layer, Meas. Sci. Technol., 8, 1553-1561, 1997.

Cenedese, A. and Moroni, M., and Querzoli, G.: Application of PTV to the Study of Penetrative Convection, 10th International Symposium on Flow Visualization, Kyoto (Japan) August 2002, 2005. 
Cushman, J. H., Park, M., Kleinfelter, N., and Moroni, M.: Superdiffusion via Lévy lagrangian velocity processes, Geophys. Res. Lett., 32, L19816, 2005.

Deardorff, J. W., Willis, G. E., and Lilly, D. K.: Laboratory investigation of non-steady penetrative convection, J. Fluid Mech., 35(1), 7-31, 1969.

Deardorff, J. W.: Convective velocity and temperature scales for the unstable planetary boundary layer and for Rayleigh convection, J. Atmos. Sci. 27, 1211-1213, 1970.

Imberger, J. and Ivey, G. N.: On the Nature of Turbulence in a Stratified Fluid. Part II: Application to Lakes, J. Phys. Oceanogr. 21, 659-680, 1991.

Kato, H. and Phillips, O. M.: On the penetration of a turbulent layer into stratified fluid, J. Fluid Mech., 37(4), 643-655, 1969.

Kleinfelter, N., Moroni, M., and Cushman, J. H.: Application of the finite-size Lyapunov exponent to particle tracking velocimetry in fluid mechanics experiments, Phys. Rev. E., 72, 056306, 2005.

Klemp, J. B., Skamarock, W. C, and Dudhia, J.: Conservative split-explicit time integration methods for the compressible nonhydrostatic equations, NCAR Technical Note, Boulder, Colorado, USA, October 2000.

Lucas, B. D. and Kanade, T.: An iterative image registration technique with an application to stereo vision, Proc. Imaging Understanding Workshop, 121-130, 1981.

Miesch, M. S., Brandenburg, A., and Zweibel, E. G.: Nonlocal transport of passive scalars in turbulent penetrative convection, Phys. Rev. E, 61(1), 457-467, 2000.

Miozzi, M.: Particle Image Velocimetry using Feature Tracking and Delauny Tessellation Proc. 12th International Symposium "Application of laser techniques to fluid mechanics", Lisbon., 2004.
Moroni, M., Cushman, J. H., and Cenedese, A.: A 3D-PTV Twoprojection Study of Pre-asymptotic Dispersion in Porous Media which are Heterogeneous on the Bench Scale, Internat. J. Engin. Sci., 41(3-5), 337-370, 2003.

Moroni, M. and Cenedese, A.: Comparison among Feature Tracking and More Consolidated Velocimetry Image Analysis Techniques in a Fully Developed Turbulent Channel Flow, Meas. Sci. Technol., 16, 2307-2322, 2005.

Nieuwstadt, F. T. M., Mason, P. J., Moeng, C. H., and Schumann, U.: Large-eddy simulation of convective boundary layer: A comparison of four computer codes, Turbulent Shear Flows 8, edited by: Dust, H. et al., Springer-Verlag, 343-367, 1992.

Querzoli, G.: A Lagrangian study of particle dispersion in the unstable boundary layer, Atmos. Environ., 30(16), 2821-2829, 1996.

Stull, R. B.: An Introduction to Boundary Layer Meteorology, Kluwer, Dordrecht, 1988.

Tomasi, C. and Kanade, T.: Detection and tracking of point features. Shape and motion from image streams: a factorization method, Carnegie Mellon University, Technical Report CMU-CS-91-132, 1991.

Townsend, A. A.: Internal waves produced by a convective layer, J. Fluid Mech., 24(2), 307-320, 1964.

Udrea, D. D., Bryanston-Cross, P. J., Moroni, M., and Querzoli, G.: Particle: Tracking Velocimetry techniques Fluid Mechanics and its Application, Kluwer Academic (The Netherlands), 279-304, 2000 . 